\title{
Scaling of the conductance in gold nanotubes
}

\author{
Miriam del Valle, ${ }^{1,2}$ Carlos Tejedor, ${ }^{1}$ and Gianaurelio Cuniberti ${ }^{2}$ \\ ${ }^{1}$ Departamento de Física Teórica de la Materia Condensada, Universidad Autónoma de Madrid, Facultad de Ciencias, \\ C-V, E-28049 Madrid, Spain \\ ${ }^{2}$ Institute for Theoretical Physics, University of Regensburg, D-93040 Regensburg, Germany
}

(Received 14 February 2006; published 10 July 2006)

\begin{abstract}
A new form of gold nanobridges has been recently observed in ultrahigh-vacuum experiments, where gold atoms rearrange to build helical nanotubes, akin in some respects to carbon nanotubes. The good reproducibility of these wires and their unexpected stability allow for conductance measurements and make them promising candidates for future applications. We present here a study of the transport properties of these nanotubes in order to understand the role of chirality and of the different orbitals in quantum transport observables. The conductance per atomic row shows a light decreasing trend as the diameter grows, which is also shown through an analytical formula based on a one-orbital model.
\end{abstract}

DOI: $10.1103 /$ PhysRevB.74.045408

PACS number(s): 73.22.-f, 73.23.-b, 73.63.Fg

\section{INTRODUCTION}

Gold is known to be a good conductor for ages but its use in nanoelectronics delivers unexpected behaviors differing from its bulk properties. Recent experiments ${ }^{1-4}$ indicate that gold nanowires exhibit fascinating ordered structures that resemble those of the by now well-known carbon nanotubes (CNTs). ${ }^{5}$

High resolution microscope images show that these wires consist of coaxial nanotubes of helical atom rows coiled around the wire axis. These wires therefore present different possible helicities as CNTs do, depending on the angle in which the atom rows rotate around the tube axis. Experimental evidence so far seems to support the fact that small chiral angles are preferred by the fabricated nanotubes. The multishell nanotubes keep the difference in the number of atom rows between adjacent shells constant, leading to a kind of magic shell-closing number which is always seven. This fact can be understood as a new inner shell arises when the radii differ in approximately $a=2.88 \AA$, the neighboring distance of bulk gold, which implies a perimeter difference $(2 \pi a$ $\sim 7 a$ ) corresponding to seven atom rows nearly parallel to each other. So single-walled nanotubes should exist with six or less atom rows, and actually one single-walled tube with five atom rows has been observed. ${ }^{3}$ With seven or more atom rows a double-walled tube should be seen, which becomes a triple-walled nanotube when more than fourteen rows are present, and so on. The good reproducibility of these wires, and their stability, ${ }^{6-9}$ which is better for those with outer tubes with an odd number of atom rows, makes them promising candidates for future applications. Ballistic conductance measurements ${ }^{4}$ help to confirm the proposed structural model through its derived electronic structure. Astonishingly enough several works hint at the fact that the conductance per atom row might decrease with the number of circumferential atoms and that the number of channels does not coincide with the number of atom rows coiling around these helical structures. ${ }^{10,11}$ In this paper, we shed new light on the theoretical understanding of this phenomenon by means of both numerical and analytical calculations.

The structure of this paper is as follows. In the next sections, we first discuss the characteristics of the topological structure taken by these tubes, followed by a description of the methods used and the computational details. The results are discussed in Sec. IV and finally our conclusions are summarized in Sec. V.

\section{STRUCTURAL PROPERTIES}

Determining the structure of gold nanotubes is fundamental in order to understand their physical properties, such as conductance quantization. Experimental evidence points out that a structural transition from the thicker, internally crystalline wires into these thinner, regular but noncrystalline ones takes place at a critical radius below $2 \mathrm{~nm}$. Lattice spacing has been measured to be almost $2.88 \AA$, the neighboring distance of gold, for all the wires with diameters between 0.5 and $1.5 \mathrm{~nm}$. We therefore take this distance as the interatomic spacing of the triangular lattice, ${ }^{12}$ as we can think of

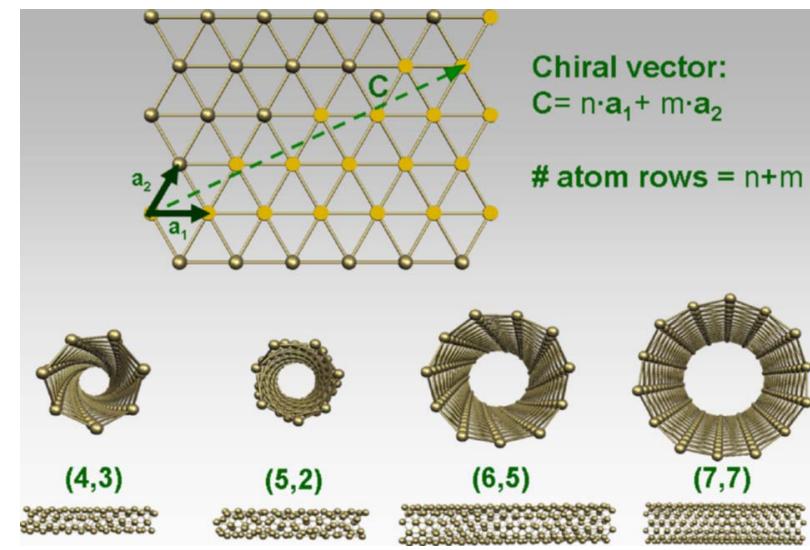

FIG. 1. (Color online) Some gold nanotubes are shown as well as the $2 \mathrm{D}$ triangular network, from which we can build these models. The coordinate system used is sketched on the lattice, the basis vectors being $\mathbf{a}_{1}$ and $\mathbf{a}_{2}$. The chiral vector $\mathbf{C}$ specifies the nanotube and corresponds to its circumference. The highlighted atoms on the lattice are those defining the region of inequivalent chiral vectors, which spans an angle of $30^{\circ}$ as can be easily understood analyzing the symmetry of the lattice. 
these cylindrical tubes as rolled-up lattice planes of fcc $\mathrm{Au}$ (111), as depicted in Fig. 1. Atoms in successive shells appear to strain a bit to maintain commensurability between inner and outer shells. Ab initio calculations show though that this shear strain should have just a very small effect on the electronic structure. ${ }^{17}$

Despite the strong intershell interactions, these structures greatly resemble those of CNTs, where the honeycomb network of carbon atoms is replaced by the triangular lattice of gold atoms. These are actually complementary networks, and the gold nanotube structure can be obtained from the one of CNTs by putting gold atoms at the center of the honeycomb framework.

We use the indices $(n, m)$ to classify the gold nanotubes (AuNTs), where $n$ and $m$ are integers defining the chiral vector, which is wrapped to form the tube. The chiral vector is then $\left(n \mathbf{a}_{1}+m \mathbf{a}_{2}\right)$, perpendicular to the tube axis, where the vectors $\mathbf{a}_{1}$ and $\mathbf{a}_{2}$ span the two-dimensional (2D) unit cell. The number of atom rows coiling around the tube axis is then $n+m$ while the nanotube translational vector reads $\mathbf{T}(n, m)=\left[(2 m+n) / d_{R}\right] \mathbf{a}_{1}-\left[(2 n+m) / d_{R}\right] \mathbf{a}_{2}, \quad d_{R}$ being the greatest common divisor of $(2 m+n)$ and $(2 n+m)$, as $\mathbf{T}$ should be the smallest lattice vector in its direction. Due to the symmetry of the triangular lattice the chiral vectors of all inequivalent tubes are comprised in a $30^{\circ}$ angle, that is, in a one-twelfth irreducible wedge of the Bravais lattice. Consequently, without losing generality we will restrict to indices with $n>0$ and $0<m<n$. Tubes of the form $(n, 0)$ and $(n, n)$ are achiral, presenting thus no handedness. Only wires with an even number of strands may have the structure $(n, n){ }^{18}$

For a fixed $n$, the radius is minimized for higher values of $m$ (string tension decreases with shrinking radius), so as to fulfill simultaneously the tightest external packing and minimal wire radius. ${ }^{9}$ The largest $m$ for an odd number of atom rows is $n-1$, leading always to a finite chirality and thus a helical structure in these cases. The outer tubes with an even number of atom rows seem to take nonhelical structures with shorter periods. The helicity of the tubes is observed experimentally through a wavy modulation of the STM images. ${ }^{2,3}$

\section{METHOD}

We study the transport properties of several of these AuNTs using a multiorbital tight-binding Hamiltonian and Green function techniques. In particular, we adopt a Slater-Koster-type ${ }^{19}$ tight-binding approach, with the parameters taken from the nonorthogonal parametrization of Papaconstantopoulos. ${ }^{20}$ Our approach remains of a microscopic nature since the symmetries of the atomic $s, p$, and $d$ valence orbitals are taken into account. Ab initio studies including shell effects could be needed for greater quantitative accuracy.

As a result, we analyze the differences in the transport calculations that arise from (i) the inclusion of all outer $s, p$, and $d$ orbitals and from (ii) the simplified model of $s$ orbitals, which is a tempting approximation for these kinds of systems. In order to compare results, we will thus use both a one-orbital Hamiltonian describing just the $s$ orbitals, and a

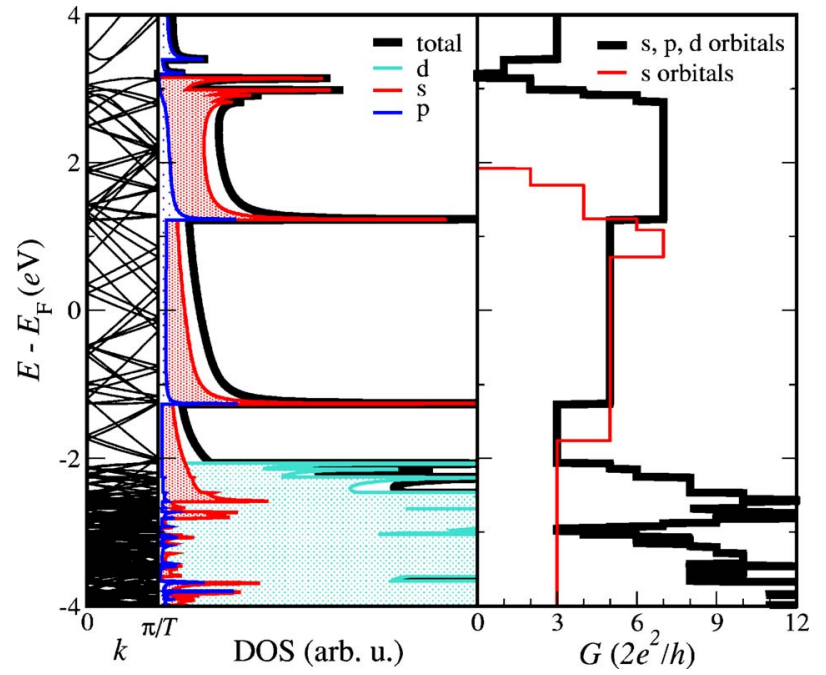

FIG. 2. (Color online) One-dimensional band structure, density of states, and conductance for the $(4,3)$ gold nanotube. For this tube the translational vector is $T=|\mathbf{T}|=3.03 \mathrm{~nm}$. The energies are shifted to set the origin at the Fermi energy, as in the next figures.

nine-orbital Hamiltonian, containing $s, p$, and $d$ orbitals including all conduction electrons.

The Hamiltonian describing our systems can be written as follows:

$$
\mathcal{H}=\sum_{\langle i, j\rangle, \alpha, \beta} H_{i \alpha, j \beta} c_{i \alpha}^{\dagger} c_{j \beta},
$$

where the indices $\langle i, j\rangle$ indicate nearest neighbor atom sites and the indices $\alpha, \beta$ mark the different orbitals. The transfer integrals $H_{i \alpha, j \beta}$ are the onsite energies or hopping parameters, dependent on whether $i$ equals $j$ or not.

The conductance is calculated from the transmission values applying the Landauer formula, using Green function techniques. ${ }^{21}$ In particular, we derive the elastic linear response conductance via the Fisher-Lee formula for the quantum mechanical transmission: ${ }^{22} G=\frac{2 e^{2}}{h} \operatorname{Tr}\left\{\boldsymbol{\Gamma}_{\mathrm{L}} \mathcal{G} \boldsymbol{\Gamma}_{\mathrm{R}} \mathcal{G}^{\dagger}\right\}$, where $\boldsymbol{\Gamma}_{\mathrm{L} / \mathrm{R}}=i\left(\boldsymbol{\Sigma}_{\mathrm{L} / \mathrm{R}}-\boldsymbol{\Sigma}_{\mathrm{L} / \mathrm{R}}^{\dagger}\right), \boldsymbol{\Sigma}_{\mathrm{L} / \mathrm{R}}$ is the self-energy of the left or right lead, respectively, and $\mathcal{G}$ is the Green function of the central region dressed by the electrodes. The wave functions of the nanotubes are assumed to extend to the leads, allowing us to remain in a phase-coherent regime.

We consider semi-infinite single-walled nanotubes (SWNTs) as leads. To deal with such semi-infinite leads, we adopt a recursive renormalization procedure already described in Ref. 23. Considering the leads as bulk gold will yield an increased imaginary part of the lead self-energies, which will slightly decrease the conductance values. For multiwalled nanotubes (MWNTs), it has been suggested that the conductance $G$ is mainly determined by the outer shell which is also responsible for the structural stability. ${ }^{24} \mathrm{~A}$ slight reduction of the conductance is expected from finite size effects.

To include temperature effects a generalization of the Landauer approach is followed as introduced earlier by Bagwell and Orlando, ${ }^{25}$ giving the finite temperature elastic coherent conductance as a convolution of the zero temperature 


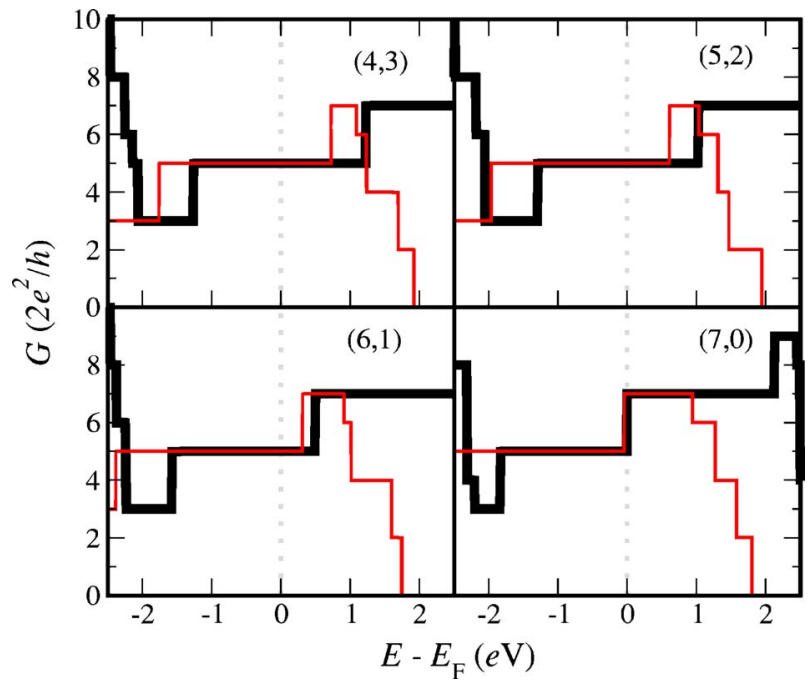

FIG. 3. (Color online) Conductance of AuNTs with seven atom rows but different chiralities. Thin lines indicate results including $s$ orbitals only, whereas the thicker ones are showing the calculations with all nine outer orbitals.

conductance and the thermal smearing function $-\frac{\partial f(E)}{\partial E}$ $=\frac{1}{4 k_{\mathrm{B}} T} \operatorname{sech}^{2}\left(\frac{E}{2 k_{\mathrm{B}} T}\right), f$ being the Fermi distribution. This convolution is a natural way to incorporate finite temperature effects leading to a broadening of the energy levels, as it averages the conductance over an energy range $k_{\mathrm{B}} T$.

\section{RESULTS AND DISCUSSION}

The calculated density of states and the conductance for the gold nanotube $(4,3)$ are shown in Fig. 2. This tube is one of the simplest possible candidates with seven atom rows around its circumference (Fig. 1). The colored areas show the contribution of the different orbitals to the total density of states, indicating a dominance of $s$ orbitals around the Fermi level. Considering only $s$ orbitals around the Fermi energy is thus a good approximation, but when applying a bias it can be critical to include all $s, p, d$ outer orbitals, as they play an important role in the opening of new conduction channels.

A comparison of the conductance of nanotubes of the same diameter but different chiralities, as shown in Fig. 3, suggests that no significant difference is expected in their conductance values at the Fermi energy. For applied bias voltages the current will show a chirality dependence, reflecting the different energies at which scattering states open.

In Fig. 4 the conductance is plotted for nanotubes of different radii, chosen as to have the smallest chirality possible for a given radius. The number of atom rows around the nanotube axis in each of them is $6,7,9,11,13$, and 14 , respectively. We see that, though the conductance increases for thicker nanotubes, this is not the general trend if divided by the number of atom rows. We can observe again how the expansion of the orbital basis to include all conduction electrons changes the energies at which new channels open, being critical to obtain correct current values.

By analyzing the Brillouin zone of the two-dimensional gold lattice taking into account only $s$ orbitals, one can easily

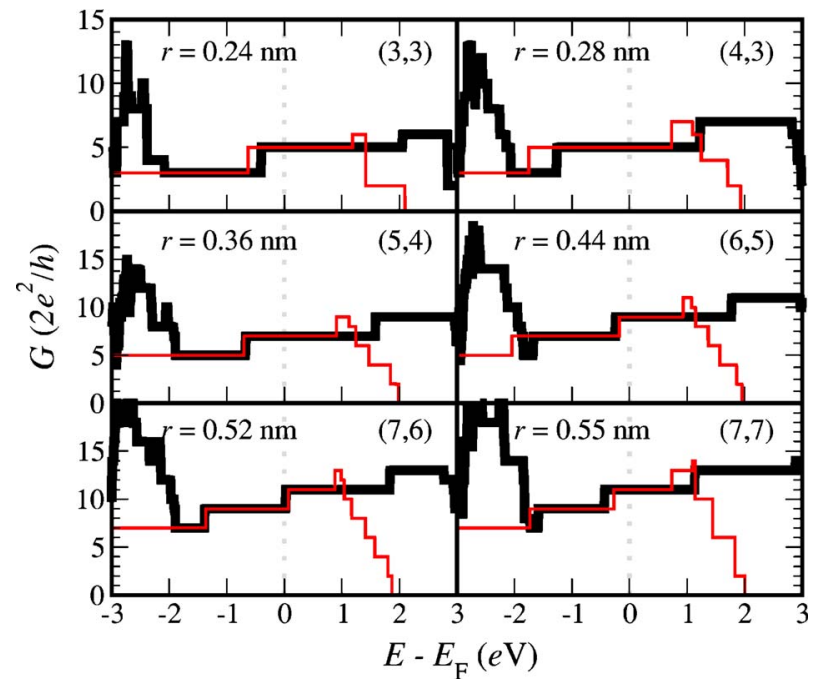

FIG. 4. (Color online) Conductance of AuNTs with different radii $r$. Thin lines indicate results including $s$ orbitals only, whereas the thicker ones are showing the calculations with all nine outer orbitals.

demonstrate after studying its dispersion relation that the Fermi surface is with great accuracy approximated by a circle with a radius close to $k_{\mathrm{F}}=2 \sqrt{\pi} /(\sqrt[4]{3} a)$, where $a$ $=2.88 \AA$. This simple model allows us to have an analytical approach to the conductance, as the number of energy bands crossing the Fermi surface, since the Brillouin zone of the gold nanotubes consists of parallel line segments due to the quantization of the wave vector along the circumferential direction. For simplicity we can restrict this counting to the first Brillouin zone by folding back to this area the segments lying outside it, which will elongate the lines inside it, as

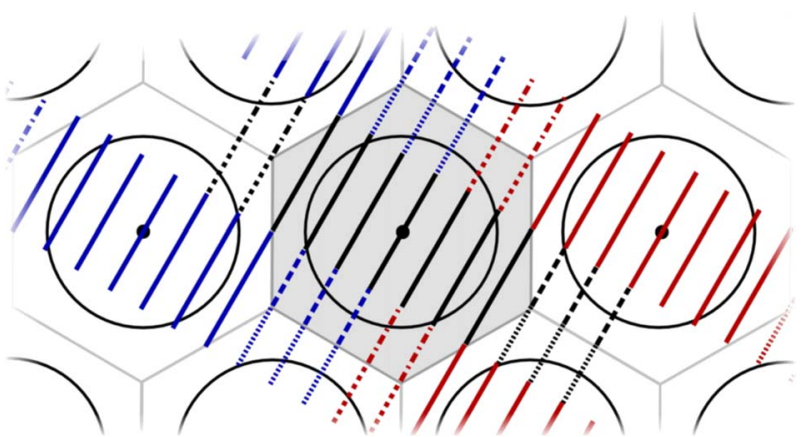

FIG. 5. (Color online) Schematic illustration of the reciprocal lattice of the 2D hexagonal Bravais lattice of $\mathrm{Au}$ (111) where the first Brillouin zone is highlighted through a darker background. The $\mathbf{k}$ vectors lying at the Fermi surface give rise to a nearly perfect circle in this $2 \mathrm{D}$ structure. The thicker cutting lines represent the Brillouin zone of a nanotube in an extended zone scheme which allows the use of the dispersion relation calculated for the 2D gold layer to get the bands of a folded nanotube. In this example we show the wave vectors giving the bands in a $(6,0)$ AuNT, a good candidate for this visualization as it has a small number of bands. These lines are plotted as solid bands inside the first Brillouin zone, and are plotted thinner when folded back into it through the reciprocal lattice vectors of the gold layer. 


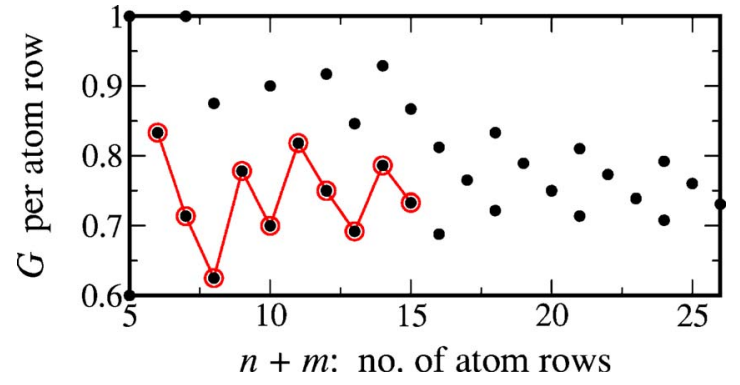

FIG. 6. (Color online) The conductance per atom row at the Fermi energy based on the analytical model accounting only for $s$ orbitals described in the text is shown for all possible $(n, m)$ NTs, reproducing exactly the values of the numerical calculations. The solid line highlights the AuNTs with the smallest chiralities seen in the experiments.

pictured in Fig. 5 (Ref. 26). The conductance can then be written as follows:

$$
G=\frac{2 e^{2}}{h}\left[2 \operatorname{Int}\left(\sqrt{\frac{n^{2}+m^{2}+n m}{\pi \sqrt{3}}}\right)+1\right]
$$

and is only dependent of the nanotube indices and not on hopping parameters or onsite energies. This model yields a perfect matching with the numerical calculations described before. The result of this approximation of the conductance at the Fermi level is plotted in Fig. 6 for nanotubes of different radii and chiralities, presenting the conductance per atom row. The solid line in this figure marks the results of AuNTs with the smallest helicity as characterized in Fig. 4. All these tubes present a number of channels smaller than the number of coiling atom rows, which is thus a characteristic of the geometry and electronic states of the nanotubes. We can observe how the upper envelope of all these points slowly decreases with an increasing number of coiling atom rows, or likewise with an increasing diameter. The conductance values will nevertheless be reduced by considering more realistic gold leads. This has also been confirmed in first-principles calculations. ${ }^{11}$

Gold nanotubes have been observed as short $(L \sim 4 \mathrm{~nm})$ conductors between bulk gold electrodes. This calls for an extension of our theory to account for finite size effects. We did this by parametrically introducing into the nanotube two tunneling barriers at a distance $L$. Figure 7 shows the conductance between two homologous semi-infinite electrodes which are taken into account through modified self-energies. This modification is introduced by a multiplicative factor $\alpha$, which simulates the barriers. Finite size effects can be made equally apparent by considering wide band leads, i.e., $\Sigma_{W B}(E) \cong-i \operatorname{Im} \Sigma\left(E=E_{\mathrm{F}}\right)$ to simulate bulk gold electrodes. As we can see in Fig. 7, we obtain the same kind of oscillating behavior with both approximations. This shows that the results of our model provide an upper limit for the conductance.

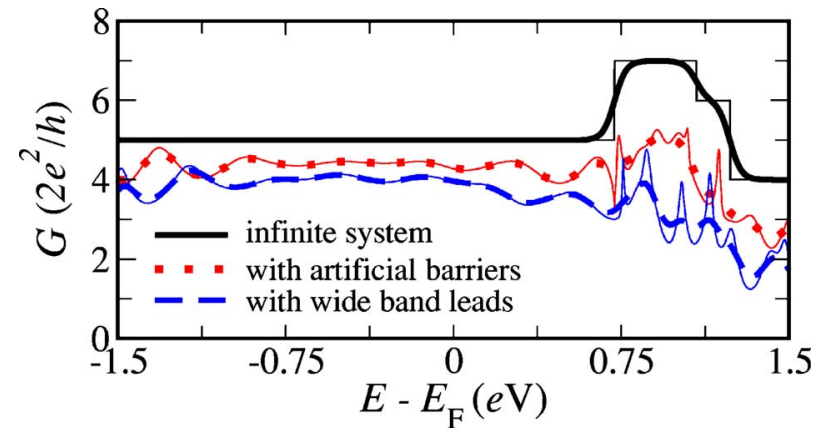

FIG. 7. (Color online) Conductance of the $(4,3)$ AuNT for a perfect infinite NT, for a finite tube with barriers in the connecting regions to the electrodes (where the constant $\alpha$ is introduced in the text equals 0.6) and for a finite tube as in the previous case but in the wide band limit approximation taken at the Fermi energy. The lines corresponding to the last two cases of oscillations, show due to scattering at the ends of the finite size tube. The thicker lines correspond to room temperature conductance ${ }^{25}$ while the thin lines underlying them correspond to zero temperature conductance.

\section{CONCLUSIONS}

In conclusion, we investigated the electronic properties of several gold nanotubes. We built the Hamiltonians of the systems by applying a tight-binding model for $s$ orbitals as well as for all outer orbitals. All AuNTs are equally metallic independent of their chirality or diameter in contrast to the results obtained for their carbon counterparts, ${ }^{27}$ but expected from gold electronic nature. In the case of gold the quantization of electron waves along the circumference of the tube results in a Brillouin zone composed of line segments that always cut the Fermi surface of the $\mathrm{Au}$ [111] layer due to the continuity of this surface.

The $s$-orbital calculations demonstrate that a one-orbital approach to the problem of gold nanotubes is an excellent approximation for energies around the Fermi level, but should be avoided when applying a finite bias voltage to the system.

An analytical formula for the conductance of the $s$-orbital calculations, matching the numerical results perfectly, shows that the number of conduction channels is smaller than the number of atom rows and that there is a slight decrease of the conductance per atom row as the radius increases, though remains rather constant for the tubes of smallest helicity.

\section{ACKNOWLEDGMENTS}

We are grateful to Yoshifumi Oshima for making us aware of his experimental data prior to publication, and to Pasquale Pavone for helpful hints to improve the appearance of the graphics. This work was partially funded by the Volkswagen Foundation (Germany) under Grant No. I/78 340, by the EU grant CARDEQ under Contract No. IST-021285-2, by the MEC (Spain) under Contract Nos. MAT2005-01388, NAN2004-09109-CO4-04, and by the CAM under Contract No. S-0505/ESP-0200. M.D. acknowledges the support from the FPI Program of the Comunidad Autónoma de Madrid. 
${ }^{1}$ Y. Kondo and K. Takayanagi, Phys. Rev. Lett. 79, 3455 (1997).

${ }^{2}$ Y. Kondo and K. Takayanagi, Science 289, 606 (2000).

${ }^{3}$ Y. Oshima, A. Onga, and K. Takayanagi, Phys. Rev. Lett. 91, 205503 (2003).

${ }^{4}$ Y. Oshima, K. Mouri, H. Hirayama, and K. Takayanagi, J. Phys. Soc. Jpn. 75, 053705 (2006).

${ }^{5}$ S. Reich, C. Thomsen, and J. Maultzsch, Carbon Nanotubes (Wiley-VCH, Berlin, 2004); R. Saito, G. Dresselhaus, and M. S. Dresselhaus, Physical Properties of Carbon Nanotubes (Imperial College Press, London, 1998); E. Thune and C. Strunk, Introducing Molecular Electronics (Springer, Berlin and Heidelberg, 2005), Chap. 13.

${ }^{6}$ O. Gülseren, F. Ercolessi, and E. Tosatti, Phys. Rev. Lett. 80, 3775 (1998).

${ }^{7}$ G. Bilalbegović, Phys. Rev. B 58, 15412 (1998).

${ }^{8}$ E. Z. da Silva, A. J. R. da Silva, and A. Fazzio, Phys. Rev. Lett. 87, 256102 (2001).

${ }^{9}$ E. Tosatti, S. Prestipino, S. Kostlmeier, A. Dal Corso, and F. Di Tolla, Science 291, 288 (2001).

${ }^{10}$ R. T. Senger, S. Dag, and S. Ciraci, Phys. Rev. Lett. 93, 196807 (2004).

${ }^{11}$ T. Ono and K. Hirose, Phys. Rev. Lett. 94, 206806 (2005).

${ }^{12}$ Also boron nanotubes (Ref. 13) have an underlying triangular lattice. Still, they do exhibit a puckered surface which is shown to be an important stabilizing factor (Ref. 14). Although their metallicity have been predicted (Refs. 15 and 16), transport measurements are not yet available.
${ }^{13}$ D. Ciuparu, R. F. Klie, Y. Zhu, and L. Pfefferle, J. Phys. Chem. B 108, 3967 (2004).

${ }^{14}$ I. Boustani, A. Rubio, and J. A. Alonso, Chem. Phys. Lett. 311, 21 (1999).

${ }^{15}$ A. Quandt and I. Boustani, ChemPhysChem 6, 2001 (2005).

${ }^{16}$ M. H. Evans, J. D. Joannopoulos, and S. T. Pantelides, Phys. Rev. B 72, 045434 (2005).

${ }^{17}$ X. Yang and J. Dong, Phys. Rev. B 71, 233403 (2005); C.-K. Yang, Appl. Phys. Lett. 85, 2923 (2004).

${ }^{18}$ We can decode our notation $(n, m)$ into the one used in parallel with lattice vectors spanning an angle of $120^{\circ}$ such as $(n$ $+m, n)$ for an equivalent AuNT for transport.

${ }^{19}$ J. C. Slater and G. F. Koster, Phys. Rev. 94, 1498 (1954).

${ }^{20}$ D. A. Papaconstantopoulos, Handbook of the Band Structure of Elemental Solids (Plenum, New York, 1986).

${ }^{21}$ G. Cuniberti, F. Grossmann, and R. Gutiérrez, Adv. Solid State Phys. 42, 133 (2002).

${ }^{22}$ D. S. Fisher and P. A. Lee, Phys. Rev. B 23, 6851 (1981).

${ }^{23}$ M. del Valle, C. Tejedor, and G. Cuniberti, Phys. Rev. B 71, 125306 (2005).

${ }^{24}$ A. Hasegawa, K. Yoshizawa, and K. Hirao, Chem. Phys. Lett. 345, 367 (2001).

${ }^{25}$ P. F. Bagwell and T. P. Orlando, Phys. Rev. B 40, 1456 (1989).

${ }^{26}$ W. A. Harrison, Phys. Rev. 118, 1190 (1960).

${ }^{27}$ R. Saito, M. Fujita, G. Dresselhaus, and M. Dresselhaus, Appl. Phys. Lett. 60, 2204 (1992); N. Hamada, S. I. Sawada, and A. Oshiyama, Phys. Rev. Lett. 68, 1579 (1992). 